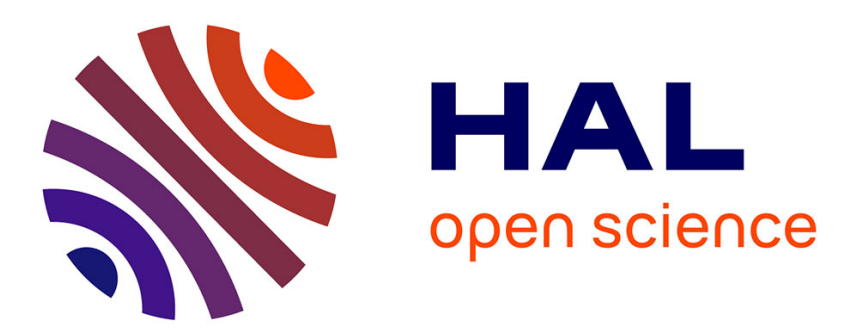

\title{
Domain decoration in dipolar coupled ferromagnetic stacks with perpendicular anisotropy
}

S. Wiebel, J. P. Jamet, N. Vernier, A. Mougin, J. Ferré, Vincent Baltz, B. Rodmacq, B. Dieny

\section{- To cite this version:}

S. Wiebel, J. P. Jamet, N. Vernier, A. Mougin, J. Ferré, et al.. Domain decoration in dipolar coupled ferromagnetic stacks with perpendicular anisotropy. Applied Physics Letters, 2005, 86, pp.142502. 10.1063/1.1897845 . hal-01683694

\section{HAL Id: hal-01683694 \\ https://hal.science/hal-01683694}

Submitted on 25 May 2019

HAL is a multi-disciplinary open access archive for the deposit and dissemination of scientific research documents, whether they are published or not. The documents may come from teaching and research institutions in France or abroad, or from public or private research centers.
L'archive ouverte pluridisciplinaire HAL, est destinée au dépôt et à la diffusion de documents scientifiques de niveau recherche, publiés ou non, émanant des établissements d'enseignement et de recherche français ou étrangers, des laboratoires publics ou privés. 


\title{
Domain decoration in dipolar coupled ferromagnetic stacks with perpendicular anisotropy
}

\author{
S. Wiebel, ${ }^{\text {a) }}$ J.-P. Jamet, N. Vernier, A. Mougin, and J. Ferré \\ Laboratoire de Physique des Solides, UMR CNRS 8502, Université Paris Sud, 91405 Orsay, France \\ V. Baltz, B. Rodmacq, and B. Dieny \\ SPINTEC, URA CNRS/CEA 2512, CEA-Grenoble, 38054 Grenoble Cedex 9, France
}

(Received 29 December 2004; accepted 21 February 2005; published online 29 March 2005)

\begin{abstract}
The dipolar stray field effects between two nonuniformly magnetized ferromagnetic Co/Pt stacks with perpendicular anisotropy are investigated by polar magneto-optical Kerr effect microscopy. Decoration of a reversed domain in the hard stack by a domain ring in the soft stack is evidenced and interpreted by magnetostatic calculations. Mirrored $360^{\circ}$ domain walls are strongly stabilized by these interactions. (C) 2005 American Institute of Physics. [DOI: 10.1063/1.1897845]
\end{abstract}

Thin-film structures involving a soft and a hard ferromagnetic layers separated by a metallic or an insulating nonmagnetic spacer are widely used in spintronic devices for their giant magnetoresistance or tunnel magnetoresistance properties. Tremendous efforts are put toward the minimization of undesirable magnetic interactions between the layers. Among the interlayer magnetic interactions, the RudermanKittel-(Kasuya)-Yosida (RKKY) oscillatory coupling between two magnetically saturated pinhole free metallic layers has been thoroughly investigated. ${ }^{1-3}$ Correlated roughness of the ferromagnet-spacer interfaces may result in an additional conformal dipolar interlayer coupling, also called orange peel (OP) Néel effect. ${ }^{4-6}$

Dipolar stray fields play a major role in nonuniformly magnetized films. They allow explaining why a tunnel junction memory may be progressively erased, since the hard layer demagnetizes with repeated reversal of the soft layer by field cycling. This phenomenon results from the replication of the soft layer domain walls within the hard layer and vice versa. ${ }^{7}$ Even under moderate fields, mirrored domains form in the hard and soft layers. ${ }^{8}$ The stray field created by Néel walls in one layer lowers the nucleation field in the other one. ${ }^{9,10}$

Up until now, most of the studies on interlayer interactions have been investigated in systems with in-plane magnetic anisotropy and non-negligible interlayer exchange coupling. However, the increasing interest for systems with perpendicular magnetic anisotropy ${ }^{11,12}$ raises questions on the role of interlayer interactions. Such systems are promising candidates for the design of new Spintronic devices ${ }^{13,14}$ or multilevel recording media. ${ }^{15}$ Perpendicular two-state systems are attractive since they are expected to improve the density, the stability, and the reliability of magnetic spin valve or tunnel junction elements, and recording media. ${ }^{13}$

In this letter, we consider two $\mathrm{Co} / \mathrm{Pt}$ ferromagnetic multilayer stacks with perpendicular anisotropy separated by a nonmagnetic Pt layer. We demonstrate how the magnetization reversal of the soft magnetic stack is influenced by the magnetic domain state in the hard one through interlayer magnetostatic interactions. The magnetic domain pattern is probed by polar Kerr microscopy in both layers, allowing the investigation of domain nucleation and wall propagation. ${ }^{16}$

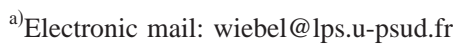

We report on the decoration of a hard magnetic domain by a ring of opposite magnetization in the soft layer due to the magnetic stray field generated by the domain in the hard layer.

We have investigated a $S_{H} / \mathrm{Pt}(4 \mathrm{~nm}) / S_{S} / \mathrm{Si} / \mathrm{SiO}_{2}$ film structure, consisting of a hard and a soft ferromagnetic Co/Pt multilayer stack, $S_{H}:[\mathrm{Pt}(1.8 \mathrm{~nm}) / \mathrm{Co}(0.6 \mathrm{~nm})]_{4}$ and $S_{S}:[\mathrm{Co}(0.6 \mathrm{~nm}) / \mathrm{Pt}(1.8 \mathrm{~nm})]_{2}$, separated by a Pt spacer layer. They were dc sputtered on a $\mathrm{Si} / \mathrm{SiO}_{2}$ substrate at room temperature under $2.5 \times 10^{-3}$ mbar Ar pressure, with deposition rates of about $0.05 \mathrm{~nm} / \mathrm{s}$. Within a given stack, the $\mathrm{Pt}(1.8 \mathrm{~nm})$ layers ensure a sufficient ferromagnetic exchange coupling $\left(H_{\mathrm{ex}} \approx 100 \mathrm{Oe}\right)$ between the Co layers and promote perpendicular anisotropy. ${ }^{17}$ The $\operatorname{Pt}(4 \mathrm{~nm})$ layer is thick enough to reduce drastically the exchange between $S_{S}$ and $S_{H}$, so that RKKY coupling across the spacer layer can be neglected. ${ }^{6}$

As expected, the polar Kerr rotation hysteresis loop in the perpendicular applied field (Fig. 1) shows two successive jumps corresponding to the coercivities of the $S_{S}\left(H_{C}^{S}\right.$ $=106 \mathrm{Oe})$ and $S_{H}\left(H_{C}^{H}=320 \mathrm{Oe}\right)$ stacks. A residual bias field, $H_{\text {op }}=8 \mathrm{Oe}$, is determined from the shift of the $S_{S}$ minor loop (Fig. 1). The latter results from the OP magnetostatic coupling that favors a parallel alignment of the magnetization in both stacks. ${ }^{6}$ The magnetic domain structure has been investigated by high-resolution $(0.4 \mu \mathrm{m})$ polar Kerr microscopy with green light at room temperature.

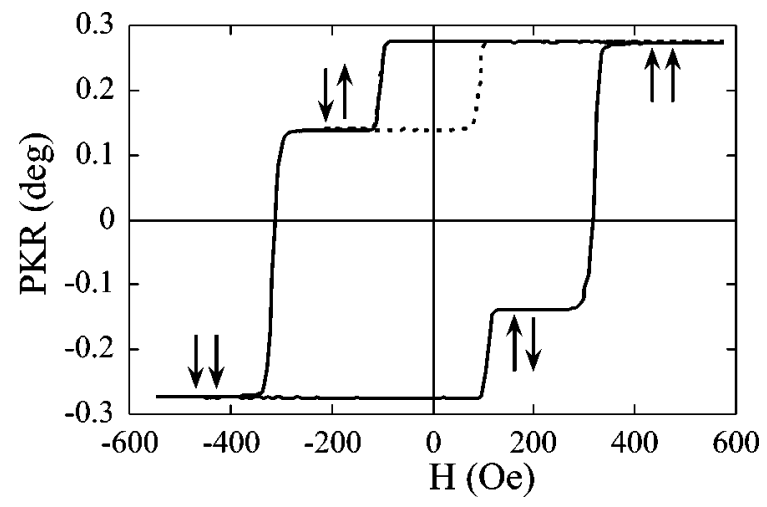

FIG. 1. Room-temperature polar Kerr rotation hysteresis major loop (straight line) and minor loop (dashed line) of the studied film structure. The field sweeping rate was $500 \mathrm{Oe} / \mathrm{s}$. 


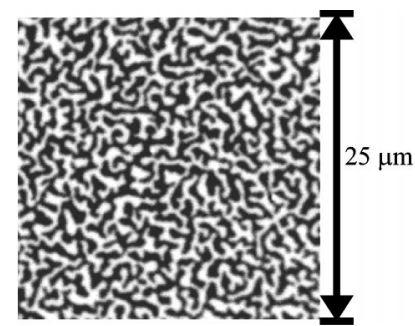

FIG. 2. Polar Kerr image of an ac-demagnetized film.

The ac-demagnetized sample state shows a ribbonlike up-up (white) and down-down (black) domain structure, specific to films with a perpendicular easy magnetization axis in the presence of magnetostatic interactions (Fig. 2). The absence of intermediate magnetization states is direct proof for a net ferromagnetic interstack interaction that provokes a perfect matching of domains and walls in $S_{S}$ and $S_{H}$.

In order to directly visualize the effect of magnetostatic interactions on field-induced remnant states, the following experimental procedure was used. After saturating the sample in the up-up spin state under a large positive field $\left(\mathrm{H}_{S}=560 \mathrm{Oe}\right)$, a negative field $H_{1}$ was applied to: (i) entirely reverse the magnetization of $S_{S}$, and (ii) create a small spin down domain in $S_{H}$ (Fig. 3). Just after switching off $H_{1}$, a white up-up magnetized decoration ring appears which expands rapidly over a short distance $(\approx 1 \mu \mathrm{m})$ [Fig. 3(a)] and also fills the $360^{\circ}$ domain walls inside the domain. In the subsequent remnant state, a spin-down $S_{S}$ domain perfectly matches the initially formed spin-down domain in $S_{H}$ (black).

The most intriguing feature here is the up-up magnetized ring decorating the central down-down domain even without an external field [Fig. 3(a)]. Note that its internal wall is rough as it is strongly pinned just below the wall in $S_{H}$. Due to the weaker wall pinning in the thinner multilayer stack, the external ring wall is smoother. Apart from the inside of the decorating ring, the $S_{S}$ magnetization points down, providing a down-up gray state far away from the $S_{H}$ domain.

As already pointed out, ${ }^{18}$ the combination of few pinning defects favors magnetically nonreversed spin-up channels (so-called $360^{\circ}$ walls) in the inside of the $S_{H}$ domain during its growth [Fig. 3(a)]. As we shall demonstrate later, the up-up spin configuration is strongly stabilized in these $S_{H}$ and $S_{S}$ matched channels. The apparent width of the up-up
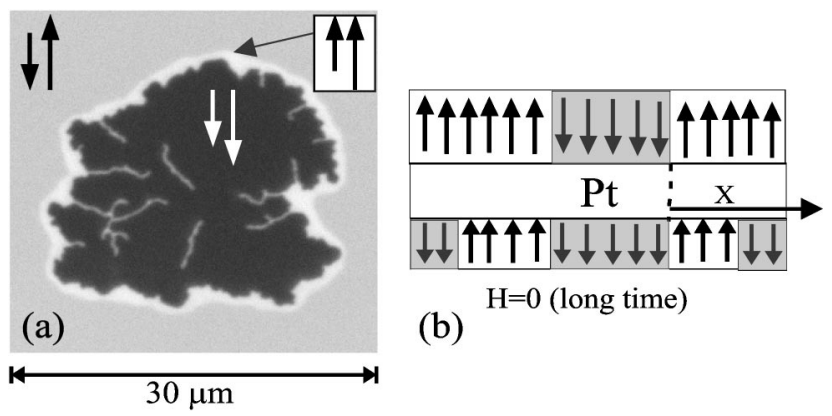

(b)

$\mathrm{H}=0$ (long time)

FIG. 3. Magneto-optical Polar Kerr rotation image (a) and sketch of the magnetization arrangement (b). The spin-down $S_{H}$ domain (black) was created by ten successive perpendicular field pulses of $50 \mathrm{~ms}$ duration and $H_{1}=-170$ Oe amplitude. The image was taken 2 min after switching off the field. hard stack, this coupling accounts for the formation and the
Downloaded 29 Mar 2005 to 129.175.97.14. Redistribution subject to AlP license or copyright, see http://apl.aip.org/apl/copyright.jsp

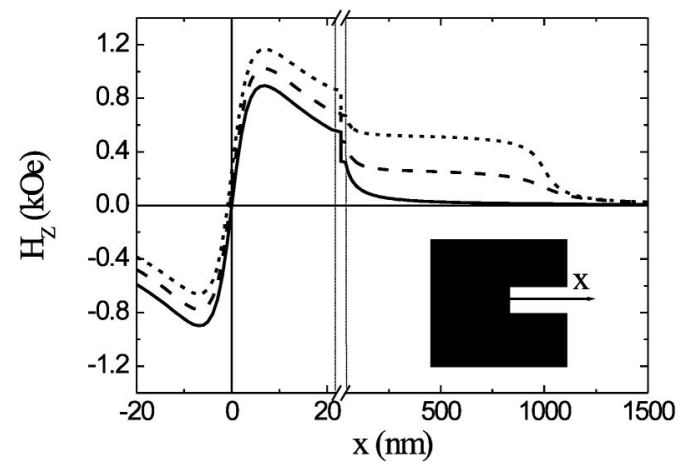

FIG. 4. Dependence of the perpendicular stray field component $H_{z}$ on the distance $x$ from the border of the $S_{H}$ domain or the base of channels with different width $e$ (straight line: No channel; dashed: $e=0.2 \mu \mathrm{m}$; dotted: $e$ $=0.1 \mu \mathrm{m})$. Inset: Sketch of the calculated structure.

magnetized channels is fixed by our optical resolution, their effective width being far smaller than $0.4 \mu \mathrm{m}$.

To be more quantitative, the evolution of the perpendicular component of the resulting stray field, $H_{\mathrm{st}}=H_{\mathrm{op}}+H_{z}\left(H_{z}\right.$ : intrastack plus interstack dipolar fields) acting inside the $S_{S}$ plane at a distance $x$ from the $S_{H}$ domain border or from the base of a $1 \mu \mathrm{m}$ long channel have been evaluated [Fig. 3(b)]. Calculations have been performed for two superimposed $20 \mu \mathrm{m}$ square down magnetized domains in $S_{S}$ and $S_{H}$, and for nonreversed spin-up channels in $S_{H}$ with varying width $e$. $H_{z}$ can reach locally high values $\left(H_{z}=800\right.$ Oe for $\left.x \approx 8 \mathrm{~nm}\right)$, just below the border of the $S_{H}$ domain [Fig. 4(a)]. Large stray field values $\left(H_{z}>400 \mathrm{Oe}\right)$ act only over a restricted width $(\approx 30 \mathrm{~nm})$ in $S_{S}$, as compared to the domain wall width $\Delta=17 \mathrm{~nm}$. Associated with the fact that domain creation needs to overcome the wall bending energy, $H_{z}$ has to be large enough over extended regions $(>100 \mathrm{~nm})$ for initiating any reversed spin-up domain in $S_{S}$. Thus, we found experimentally that the nucleation takes place first inside channels, since large stray fields $H_{z}$ act here all over the channel length (Fig. 4).

As soon as a spin-up state nucleates in $S_{S}$ in an open channel, it propagates rapidly to form a decoration ring around the $S_{H}$ domain (Fig. 3). In counterpart, since $H_{z}$ is highly negative just below the $S_{H}$ domain (Fig. 4), the corresponding spin-down domain state in $S_{S}$ becomes highly stabilized.

The decorating up-up domain expands by propagation of the external ring wall in $S_{S}$ (Fig. 3), even in $H=0$, under the action of $H_{\mathrm{st}}$. The expansion of the up-up magnetized decoration ring slows down rapidly since the external wall is submitted to a stray field essentially determined by the decrease of $H_{z}^{\text {up }}$ as $1 / x$.

The width of the up-up spin decoration ring can be tuned by applying an additional field (Fig. 5). Provided it displays the same positive sign as $H_{\text {st }}$, even a weak field $\left(H_{2}=7 \mathrm{Oe}\right)$ helps the external ring wall to propagate [Fig. 5(a)]. Correspondingly, a negative field tends to shrink the ring [Fig. 5(b)] since the applied field competes with $H_{z}$, which is strongly enhanced when the wall approaches the $S_{H}$ domain boundary. For high negative fields $(H<-100 \mathrm{Oe})$, only channels maintain an up-up spin configuration.

We have reported on the direct observation of the role of dipolar interactions between two ferromagnetic stacks with perpendicular anisotropy. For a nonuniformly magnetized 

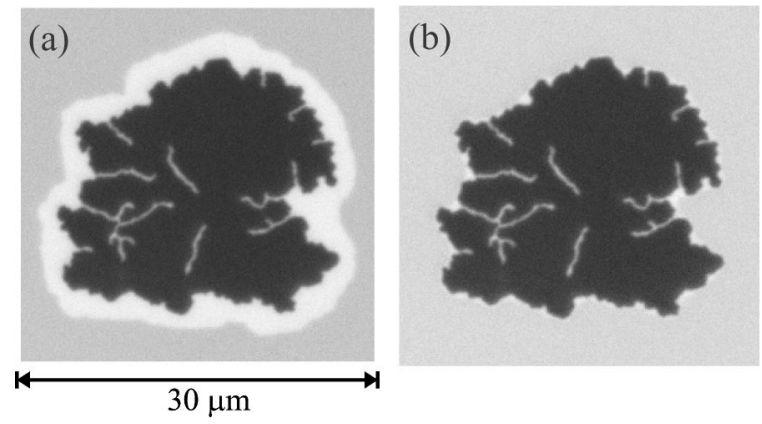

FIG. 5. Polar Kerr rotation images as in Fig. 3(a), but after applying a positive magnetic positive field $\mathrm{H}_{2}=7 \mathrm{Oe}$ during $2 \mathrm{~min}$ and freezing this state in zero field (a), or at negative field $H_{3}=-77 \mathrm{Oe}$, applied for $2 \mathrm{~min}$ (b).

propagation of domain walls within the soft stack, which gives rise to domain decoration. Our results are supported by magnetostatic calculations. In contrast to systems with inplane anisotropy, where the stray field emanates from Néel walls, here it is due to the finite domain size. Vestigial $360^{\circ}$ Bloch walls in the hard stack radiate strong fields which initiate nucleation and local propagation of reversed domains in the soft layer. Even without an external field, the walls created in the soft layer keep propagating for a long time, only driven by dipolar interactions. As for in-plane magnetized systems, stray fields can perturb the functioning of systems with perpendicular anisotropy. As in these systems lateral sizes keep shrinking, this type of magnetostatic coupling will play a fundamental role for the implementation of future devices consisting of magnetic multilayers with perpendicular anisotropy.

The authors wish to thank S. Auffret for assistance with the film deposition and hall characterization, and A. Thiaville for enlightening discussions. This letter is a part of S. Wiebel's work done as a Post-doc fellowship in the frame of the ULTRASWITCH European project (HRPN-CT-200200318). This work has been performed in part during the early stage of the MAGLOG European STREP (FP6510993) program.

${ }^{1}$ P. Bruno and C. Chappert, Phys. Rev. Lett. 67, 1602 (1991).

${ }^{2}$ S. Parkin, Phys. Rev. Lett. 67, 3598 (1991).

${ }^{3}$ V. Grolier, D. Renard, B. Bartenlian, P. Beauvillain, C. Chappert, C. Dupas, J. Ferré, M. Galtier, E. Kolb, M. Mulloy et al., Phys. Rev. Lett. 71, 3023 (1993).

${ }^{4}$ L. Néel, C.R. Acad. Sci. 255, 1676 (1962).

${ }^{5}$ B. Dieny, V. Speriosu, S. Parkin, B. Gurney, D. Wilhoit, and D. Mauri, Phys. Rev. B 43, 1297 (1991).

${ }^{6}$ J. Moritz, F. Garcia, J. Toussaint, B. Dieny, and J.-P. Nozières, Europhys. Lett. 65, 123 (2004).

${ }^{7}$ L. Thomas, M. Samant, and S. Parkin, Phys. Rev. Lett. 84, 1816 (2000).

${ }^{8}$ W. Lew, S. Li, L. Lopez-Diaz, D. Hatton, and J. Bland, Phys. Rev. Lett. 90, 217201 (2003).

${ }^{9}$ H. Fuller and D. Sullivan, J. Appl. Phys. 33, 1063 (1962).

${ }^{10}$ R. Schäfer, R. Urban, D. Ullmann, H. Meyerheim, B. Heinrich, L. Schultz, and J. Kirschner, Phys. Rev. B 65, 144405 (2002).

${ }^{11}$ O. Hellwig, A. Berger, and E. E. Fullerton, Phys. Rev. Lett. 91, 197203 (2003).

${ }^{12}$ S. Maat, K. Takano, S. Parkin, and E. Fullerton, Phys. Rev. Lett. 87, 087202 (2001).

${ }^{13}$ N. Nishimura, T. Hirai, A. Koganei, T. Ikeda, K. Okano, Y. Sekiguchi, and Y. Osada, J. Appl. Phys. 91, 5246 (2002).

${ }^{14}$ J. Sort, B. Rodmacq, S. Auffret, and B. Dieny, Appl. Phys. Lett. 83, 1800 (2003).

${ }^{15}$ V. Baltz, S. Landis, B. Rodmacq, and B. Dieny, J. Magn. Magn. Mater. 290, 1286 (2005).

${ }^{16}$ J. Ferré, Dynamics of the Magnetization Reversal, edited by B. Hillebrands and K. Ounadjela (Springer, Berlin, 2002), pp. 127-165.

${ }^{17}$ S. Landis, B. Rodmacq, and B. Dieny, Phys. Rev. B 62, 12271 (2000).

${ }^{18}$ S. Lemerle, J. Ferré, A. Thiaville, J. Chapman, P. Aitchison, S. McVitie, C. Chappert, and V. Kottler, Magnetic Hysteresis in Novel Magnetic Materials (Kluwer, Dordrecht, 1997), p. 537. 